\title{
Note sur les techniques pratiques de fabrication de l'ensilage en zone tropicale
}

\author{
par J. PAGOT, Z. DERBAL et J. LAHORE
}

\section{ENSILAGE DES FOURRAGES}

\section{1. - Généralités}

La fabrication $\mathrm{du}$ foin est rendue difficile en zone soudanienne et guinéenne par la fréquence des précipitations atmosphériques à l'époque de la floraison qui est favorable à la récolte du foin.

Il était donc naturel d'envisager l'utilisation de l'ensilage pour la constitution des réserves fourragères.

Les recherches entreprises depuis 1950 au Centre Fédéral de Recherches Zootechniques avaient pour but de préciser les techniques utilisables pour l'obtention d'un ensilage de bonne qualité.

\section{2. - Espèces à ensiler}

Parmi les fourrages spontanés et cultivés qui ont été essayés, les meilleurs résultats ont été obtenus avec les espèces suivantes dont la description est donnée en annexe :

Spontanées :

Pennisetum spp.

Panicum maximum.

Eleusine indica.

Paspalum spp.

\section{Cultivées :}

Sorghos.

Andropogon sudanensis (Sweet Sudan grass). Maïs.

Pois d'angole.

Vigna sinensis.

Canavalia ensiformis.

Mucuna spp.

Il est préférable d'associer les légumineuses aux graminées.

\section{3. - Façons culturales - Semis}

Les terres sont préparées comme pour toutes les cultures. Un labour plus ou moins profond, suivant la nature du sol, suivi d'un passage de pulvérisateur ou de cultivateur.

Les semis se font en ligne ou à la volée.

Les semis en ligne donnent des tiges plus ligneuses mais peuvent être plus facilement récoltées à l'ensileuse tractée. Ils conviennent également pour les semis de mélange graminées-légumineuses.

Les semis à la volée donnent des tiges étiolées, tendres, mais la récolte doit se faire en deux temps :

- fauchage avec une barre de coupe,

- ensuite hachage, soit avec un pik-up hachoir, soit avec un hachoir près des fosses à ensiler.

Les quantités de semences nécessaires à l'hectare sont indiquées dans le tableau suivant::

\begin{tabular}{|c|c|c|c|}
\hline $\begin{array}{c}\text { PLANTES } \\
.\end{array}$ & $\begin{array}{l}\text { SEMIS } \\
\text { EN LIGNE } \\
97 \mathrm{~cm}\end{array}$ & $\begin{array}{c}\text { SEMIS' } \\
\text { EN LIGNE } \\
40 \mathrm{~cm}\end{array}$ & $\begin{array}{c}\text { SEMIS } \\
\text { A LA VOLEE }\end{array}$ \\
\hline Sorgho indigène & $15-16 \mathrm{~kg}$ & 一 & $75-85 \mathrm{~kg}$ \\
\hline Sorgho américain & $16-18 \mathrm{~kg}$ & - & $80-90 \mathrm{~kg}$ \\
\hline Sudan grass .... & $15-20 \mathrm{~kg}$ & $25-35 \mathrm{~kg}$ & - \\
\hline Poids d'angole & $15-20 \mathrm{~kg}$ & $25-30 \mathrm{~kg}$ & $80-90 \mathrm{~kg}$ \\
\hline Crotalaires & $15-20 \mathrm{~kg}$ & $30-35 \mathrm{~kg}$ & $70-80 \mathrm{~kg}$ \\
\hline Maïs... . . & $12 \mathrm{~kg}$ & 一 & $50 \mathrm{~kg}$ \\
\hline Haricots ....... & $10 \mathrm{~kg}$ & $16-20 \mathrm{~kg}$ & - \\
\hline
\end{tabular}

\section{4. - Récolte}

A. - Ensileuse tractée (ensilage harvester) :

Les tiges sont coupées et hachées par la même 
machine. Le mélange haché est soufflé dans une remorque attelée à l'appareil à récolter.

Matériel nécessaire :

1 tracteur 35/40 CV,

I tracteur ou une jeep pour tirer les remorques,

1 ensilage Harwester,

3 remorques au minimum.

Main-d'œuvre :

2 chauffeurs,

1 aide pour surveiller le hachoir,

I manœuvre sur la remorque,

4 mancuvres pour le déchargement des remorques si ces dernières n'ont pas un système de basculage.

\section{B. - Fauchage puis hachage près des fosses :}

Les tiges coupées à la barre de coupe sont chargées en brut sur les remorques puis déchargées près des fosses où elles sont hachées. Le mélange haché est soufflé directement dans les fosses.

Matériel :

I tracteur 35 à $40 \mathrm{CV}$ avec barre de coupe,

1 tracteur ou une jeep pour tirer les remorques,

3 remorques,

1 hachoir fixe.

Main-d'œuvre :

3 chauffeurs,

8 à 10 mancuvres pour charger et décharger les remorques.

Avantages et inconvénients des 2 méthodes:

La première méthode économise de la maind'œuvre mais nécessite une mise de fonds pour un appareil qui ne travaille qu'un petit nombre de jours dans l'année.

En terrain humide, l'appareil à ensiler a tendance à s'enliser. De plus, quand les tiges sont trop mouillées, les feuilles collent contre les fouleaux et la soufflerie s'engorge.

La seconde méthode requiert une main-d'œuvre plus abondante, mais nécessite un matériel moins onéreux. Elle peut être utilisée dans une Station sans tracteur, avec une faucheuse tirée par des bœufs, le hachoir fixe pouvant fonctionner avec un moteur de ferme (type Bernard W 13).

Au Centre Fédérdl, où nous avons essayé les 2 techniques, nous donnons la préférence à la seconde.

\section{C. - Fauchage puis ensilage de l'herbe brute :}

Si on ne possède pas de hachoir, on coupe l'herbe à la faucheuse ou à la faux et on remplit les silos avec l'herbe brute.

\section{5. - Ensilage des fourrages}

Silos-fosses :

Les silos-fosses et les silos-tranchées sont très économiques.

Ceux que nous utilisons ont 1,80 a $2 \mathrm{~m}$ de profondeur sur $4 \mathrm{~m}$ de largeur et 15 à $20 \mathrm{~m}$ de longueur.

Les parois sont verticales et entretenues bien lisses. Le fond et les côtés peuvent être cimentés mais en terrain compact et bien drainé, on peut s'en dispenser. Si le terrain est très compact, on a intérêt à creuser un puisard en bout de fosse pour l'évacuation des jus.

Silos-meules :

Les simples meules de 4 à $8 \mathrm{~m}$ de diamètre donnent de meilleurs résultats que les silos-cages.

On trace les contours de la meule au diamètre voulu et on creuse à $50 / 80 \mathrm{~cm}$ de profondeur. La terre retirée servira pour recouvrir la meule.

\section{Conservateurs :}

Sel : On a obtenu d'excellents résultats en utilisant le sel à sec à raison de 4 à $5 \mathrm{~kg}$ par tonne de fourrage ensilé en meule, Si le fourrage est suffisamment humide, il est inutile 'de l'arroser. L'ensilage conservé au sel, en raison de sa saveur, est mieux appété que celui fait avec les autres conservateurs.

Acide formique : L'acide commercial à $90 \%$ est dilué à rasson d'un litre d'acide pour 201 d'eau.

Il faut utiliser 4 à 51 de solution pour $100 \mathrm{~kg}$ de légumineuses.

3 à $4 \mathrm{l}$ de solution pour $100 \mathrm{~kg}$ de fourrage spontané ou de céréales.

L'ensilage obtenu avec ce conservateur a une très bonne odeur et se conserve bien, même quand le silo est ouvert.

Il a contre lui son prix de revient assez élevé du fait du coût du transport des touries d'acides ( $327 \mathrm{fr}$ le litre à Bamako).

Sel Sovilon : Le sel Sovilon, fabriqué par les Établissements Solvay, est un chloro-bromo-iodacétate d'éthoxyglycol et d'éther oxydo do glycolpolyéthylénique, doué de propriétés bactériostatiques.

Il se présente sous forme d'une poudre cristalline conditionnée en emballage cartonné paraffiné contenant $1 \mathrm{~kg}$ de produit.

On utilise le produit à raison de $0,800 \mathrm{~kg}$ à $1 \mathrm{~kg}$ pour 10 tonnes de fourrage vert.

Le prix du sel Sovilon rendu à Barnako est de 1.198 fr CFA le kilo.

$\mathrm{Ce}$ produit est un excellent conservateur. Il a comme avantage son faible volume, son prix de revient acceptable (100 fr par tonne de fourrage à ensiler) et sa non-toxicité. 


\section{Remplissage des silos :}

Cette opération doit être effectuée rapidement et si possible à l'abri des pluies.

\section{Silos-fosses :}

Dans les fosses, l'herbe hachée est disposée en couches de $15 / 20 \mathrm{~cm}$ d'épaisseur et arrosée avec la solution de conservateur à l'aide d'un arrosoir a pomme. On tasse fortement par piétinement surtout dans les coins et sur les bords.

Quand le silo est rempli, on fait passer un tracteur pour bien tasser. Pour la dernière couche de fourrage, on double les proportions de conservateur.

On recouvre l'herbe avec une couche de paille de 10 à $20 \mathrm{~cm}$ d'épaisseur. On laisse reposer pendant 12 heures et on recouvre le tout d'une couche de terre de 40 à $50 \mathrm{~cm}$.

Les crevasses qui se produisent par la suite dans le revêtement de terre sont comblées avec de la terre humide.

\section{Silos-meules :}

Les herbes entières sont entassées par couches successives suivant les rayons de la fosse et sur une hauteur de 3 à $4 \mathrm{~m}$.

On peut utiliser tous les conservateurs indiqués précédemment.

Quand la meule est dressée, on la recouvre d'une couche de paille et on la charge avec la terre retirée pour creuser le pied de la meule.

Il y a avantage à faire plusieurs petites meules plutôt qu'une seule grande; la confection et le recouvrement sont plus faciles et les pertes après ouverture, réduites.

Dans le calcul des prix de revient on a compté que l'amortissement du matériel agricole se faisait en 5 ans; en raison du faible nombre d'heures d'utilisation par an, celui de l'ensileuse se fait en 10 ans.

Pour la campagne 1954, les prix de revient à la tonne des ensilages conservés en fosse au sel Sovilon, à l'acide formique et en meule au sel ordinaire s'établissent comme suit :

Ensilage haché au sel Sovilon:

Façon culturales........

122,75

Récolte et ensilage. . . . . 634,20

Ámortissement ensileuse. $\quad 240$

996,95

Ensilage haché à l'acide formique :

Façons culturales...... 122,75

Récolte et ensilage...... 1.174

Amortissement ensileuse. 240
Ensilage non haché en meule avec du sel ordinaire:

Fauchage à la main des

herbes de brousse. . . . 257,50

Ensilage ........... 808,80

Sel (5 $\mathrm{kg}$ par tonne).....

55

$1.121,30$

Utilisation de l'ensilage :

Les fosses à ensilage peuvent être ouvertes 3 ou 6 mois après leur remplissage. On retire la terre à l'une des extrémités et l'ensilage est prélevé par tranches verticales. La terre est retirée au fur et à mesure de la consommation.

On protège la partie découverte de l'action du soleil par un paillon.

Pour les meules, les prélèvements se font à partir du sommet par couches horizontales.

L'ensilage est distribué le soir au retour du pâturage. Les animaux ont ainsi plus de temps pour le consommer.

L'ensilage conservé à l'acide formique ou au sel Sovilon est mieux accepté s'il est arrósé avec une solution salée.

L'ensilage conservé entier et salé en meule est rapidement consommé et il n'y a aucun refus.

Au Centre Fédéral de Recherches Zootechniques, l'ensilage est distribué comme complément, l'alimentation de base étant constituée: par l'herbe consommée au pâturage dans la journée.

Les rations distribuées sont les suivantes :

\begin{tabular}{|c|c|c|}
\hline & $\begin{array}{c}\text { ENSLAGE } \\
\text { HACHÉ }\end{array}$ & $\begin{array}{c}\text { ENSILAGE } \\
\text { ENTIER } \\
\text { en meule }\end{array}$ \\
\hline Vache N'Dama & $5 \quad \mathrm{~kg}$ & $\mathrm{~kg}$ \\
\hline Taureau N'Dama .... & $5,5 \mathrm{~kg}$ & $6,650 \mathrm{~kg}$ \\
\hline Vache zébu...... & $2,7 \mathrm{~kg}$ & $5,500 \mathrm{~kg}$ \\
\hline Taureau zébu ... & $3,1 \mathrm{~kg}$ & $\mathrm{~kg}$ \\
\hline
\end{tabular}

\section{DESCRIPTION ET CARACTÉRISTIQUE DE QUELQUES FOURRAGES TROPICAUX UTHISABLES POUR L'ENSHAGE}

\section{1. - Graminées spontanées}

Pennisetum. - Les plus fréquents sont les suivants :

- Pennisetum mollissimum.

- Pennisetum setaceum.

- Pennisetum violaceum.

- Pennisetum polystachyum.

- Pennisetum pedicellatum.

Cette graminée est appelée en Bambara "N'Golo". Elle pousse autour des villages et sur les anciennes jachères. 
C'est une plante vivace qui forme des touffes serrées. Les tiges dressées ont de 0,80 à $2 \mathrm{~m}$ de hauteur. Les feuilles sont longues, assez larges.

L'inflorescence est une panicule spiciforme cylindrique de différentes couleurs, jaune, violette, rouge ou bleue suivant l'espèce et la variété.

Les Pennisetum doivent être ensilés vers le 15 septembre; l'ensilage obtenu est de bonne qualilé, il est conseillé d'associer les Pennisetum aux légumineuses.

Les Pennisetum peuvent être cultivés; on les multiplie assez bien par semis direct à la volée ou mieux en lignes et également par boutures.

Le rendement moyen en culture varie de 30 à 55 tonnes de fourrage vert à l'hectare.

Panicum maximum. - Herbe de Guinée, Guinea grass. Cette graminée est appelée en Bambara «Toba».

On la rencontre sur les sols frais aux abords des cours d'eau et dans les jachères.

Il existe de nombreuses variétés locales qui se différencient d'après la hauteur des tiges et la couleur des grains.

C'est une plante vivace formant des touffes ouvertes, les tiges solides dressées ont 1,80 à $3 \mathrm{~m}$ suivant la richesse du sol.

Les feuilles ont de 60 à $90 \mathrm{~cm}$ de longueur sur 2 à $4 \mathrm{~cm}$ de large.

L'inflorescence est une panicule ramifiée dont les graines vertes deviennent foncées à maturité.

Les Panicum peuvent être cultivés soit par semis direct en ligne ou en poquets, à $50 \mathrm{~cm}$ sur la ligne. On peut également les multiplier par repiquage d'éclats de souches.

La première coupe peut être faite 3 mois après le semis ou le repiquage. Elle repousse très vite et peut fournir 2 aे 3 coupes.

Le rendement en culture est de 30 tonnes de fourrage vert à l'hectare à la première coupe.

Après la récolte des graines, au début de novembre, on peut obtenir une repousse en coupant les chaumes à 20 à $30 \mathrm{~cm}$ du sol. On peut faucher jusqu'en décembre; la repousse est encore appréciable et les animaux la consomment volontiers.

Paspalum: - C'est une graminée rustique, perenne, qui se plait dans les endroits frais tels que les pâturages de zones basses et les anciennes jachères.

Elle résiste bien à la sécheresse.

La variété la plus commune au Soudan est $P$. scrobiculatum qui forme des touffes basses atteignant de 30 à $45 \mathrm{~cm}$ de hauteur.

Les tiges sont généralement dressées, atteignant $20-25 \mathrm{~cm}$ de longueur. Les feuilles s'étalent en largeur et couvrent vite le sol.
L'inflorescence est unilatérale, les graines ovaloorbiculaires, aplaties sur le dessus, sont vertes:

La plante porte 2 à 6 épis longs de 4 à $10 \mathrm{~cm}$.

Les animaux recherchent cette graminée surtout vers la fin de la saison des pluies, quand les autres plantes commencent à durcir. Fille résiste bien au piétinement et envahit vite les pâturages ou prairies où elle est introduite.

En ensilage, mélangée aux légumineuses et plantes riches en glucides (maïs, sorghos) elle donne d'excellents résultats.

Elle se multiplie assez bien par semis direct et mieux encore par plantation d'éclat de souches.

Pour l'ensilage, on la récolte vers la mi-septembre, au plus tard au début d'octobre.

Le rendement est moyen.

Brachiaria. - Plusieurs variétés existent au Soudan.

C'est une graminée vivace, robuste, à racines rłizomateuses, formant des touffes serrees qui atteignent 40-60 $\mathrm{cm}$ de hauteur.

L'inflorescence est une panicule unilatérale formant des épis courts qui sont pourvus de poils fauve-doré le long du rachis et de chaque côté des épis.

Les fleurs se touchent et sont placées sur une seule rangée. Elles ont 3 à $4 \mathrm{~mm}$ de longueur.

Les feuilles, longues de 10 à $20 \mathrm{~cm}$, sont larges de 5 à 20.

On la rencontre dans les savanes, dans les sols les plus variés. Elles reste longtemps verie en saison sèche dans les endroits où elle forme des touffes épaissos.

Elle est très recherchée par les animaux au début de la végétation, moins au début de la saison sèche quand ses feuilles durcissent.

Pour l'ensilage, elle peut être utilisée en mélange avec d'autres plantes et doit être récoltée au début de la floraison, vers la fin septembre.

La multiplication se fait par semis direct ou par repiquage des rhizomes qui développent de nombreuses plantes autour de la souche maternelle.

Le rendement en culture atteint son maximum la deuxième année; à l'état spontané il est moyen.

Echinochloa. - Graminée vivace, à tige dressée de 60 à $110 \mathrm{~cm}$ de hauteur, avec le sommet qui retombe et s'enracine facilement aux noeuds en formant de mouvelles touffes.

On rencontre plusieurs variétés qui se reconnaissent d'après la longueur de la panicule (de 10 à $40 \mathrm{~cm}$ ) et des épis $(2$ à $8 \mathrm{~cm}$ ) de couleurs différentes (vertes, violacées).

Elle se plait dans les terres lourdes; humides, aux abords des cours d'eau et dans les rizieres.

Certaines variétés ( $E$. crus galli et $E$. colona) poussent abondamment dans les jachères. 
Elles sont consommées par les animaux à l'etat jeune.

Pour l'ensilage, on la récolte en septembre avant le début de la floraison,

La multiplication se fait par bouture; moins bien par le semis. Elle exige une terre humide assez profonde.

C'est une excellente graminée à ensiler dans les régions rizicoles.

Eleusine. - C'est une espèce rudérale et très nitrophile qu'on rencontre autour des étables et des parcs à bétail.

Elle est annuelle, très prolifique, utilisée dans certaines régions comme céréales à graines.

Ia plante atteint 30 à $70 \mathrm{~cm}$ de hauteur; les feuilles sont longues de 20 à $40 \mathrm{~cm}$ el large de $8 \mathrm{~mm}$.

Ia variété la plus fréquente est Eleusine indica qui a une hampe florale aplatie sur toute la Iongueur, avec 3 ou 4 épis, quelquefois plus. Les fleurs sont disposées en petits racèmes de $5 \mathrm{~mm}$ appliqués les uns contre les autres. Les glumes sont lancéolées.

Elle se multiplie très bien par semis direct, en ligne ou à la volée, et se développe très rapidement.

Très appétée par les animaux comme fourrage vert, elle peut fournir facilement 2 à 3 coupes en saison des pluies. La troisieme coupe peut être ensilée vers fin décembre, en mélange avec d'autres plantes.

Le rendement en 3 coupos varie entre 30 et 50 tonnes.

\section{2. - Espèces cultivées}

Sorgho. - On utilise soit les variétés locales du sorgho sucré type Djebana et Feterita, ou les sorghos américains hâtifs.

Les premiers donnent un rendement très élevé en matière verte, mais sont généralement assez tardifs. Semés à la volée, le rendement moyen à l'hectare est de 30 tonnes.

Les sorghos américains sont très hâtifs et atteignent le maximum de leur développement en 2-3 mois. Les rendements varient entre 10 et 15 tonnes à l'hectare.

Sudan-grass, Andropogon sudanensis, sorgho à feuilles menues. C'est une graminée vivace, résistant à la sécheresse, de croissance rapide et formant des grandes touffes à larges feuilles.

Les panicules, moins fournies en graines que celles des autres sorghos; donnent des graines plus petites que le sorgho.

C'est un excellent fourrage pour l'ensilage.

On le sème d'habitude en lignes espacées de $1 \mathrm{~m}$. La récolte se fait $80-100$ jours après le semis, avant l'épiaison.
En mélange avec les graminées basses, il donne un excellent ensilage.

On peut le semer aussi en lignes rapprochées $(40 \mathrm{~cm})$ et on obtient ainsi des tiges plus fines et plus hautes. La coupe pour ensilage peut se faire un peu plus tôt, c'est-à-dire 70-80 jours après semis.

En mélange avec d'autres graminées et les légumineuses it fournit un excellent ensilage.

Maïs. - Le maïs indigène à longues tiges, récolté au début de la floraison, fournit une excellente matière à ensiler, riche en glucides, mais assez pauvre en protides. Il doit être mélangé avec de jeunes graminées et légumineuses.

Pois d'angole. - Cajanus cajan, Cajanus indicus, ambrevade (pigeon pea). C'est une excellente légumineuse africaine, très répandue, qui forme des arbustes assez hauts $(1,80-3 \mathrm{~m})$ àvec système radiculaire très étalé et profond $(40-110 \mathrm{~cm})$. Il se lignifie très vite, mais fournit une grande quantité de feuilles tres appétées par les animaux. Récolté avant la floraison, il fournit un excellent fourrage dont la valeur alimentaire est très proche de celle de la luzerne.

Il ne doit pas être ensilé seul car, trop riche en protides, il supporte mal la conservation. Mais mélangé aux plantes riches en glucides (sorgho, mais), il fournit un excellent ensilage. En culture, il fournit environ 12 tonnes de fourrage vert à l'hectare.

Mélanges fourragers. - Nous avons essayé d'associer lors des semis, les sorghos et le maïs avec les légumineuses, notamment les haricots et crotalaires. Ce mélange donne des résultats favorables et entraîne une grande économie de main-d'œuvre. La plante principale (mil-maïs) est semée en lignes et les plantes secondaires sont semées ensuite à la main entre les lignes, après le premier binage.

Crotalaires : Crotalaria retusa et juncea. Ce sont des plantes érigées, d'une croissance très rapide et qui résistent bien à l'humidité et à la sécheresse.

Les animaux les consomment assez bien au début de la végétation.

Elles produisent une quantité importante de matière verte qui peut être ensilée avec d'autres plantes. Trop riches en eau et en cellulose, elles fournissent un ensilage d'une qualité médiocre. qui se dessèche vite et devient fibreux.

Le semis se fait soit en lignes espacées de $50 \mathrm{~cm}$ soit à la volée.

Le rendement est assez faible étant donné la nécessité de couper la plante avant le début de la floraison. Il varie entre 10 et 18 tonnes à l'hectare. 


\section{Vigna, Cow-Pea :}

L'espèce la plus répandue est Vigna cajan ou, Vigna sinensis. Cette plante pousse rapidement et couvre bien le sol. De nombreuses variétés se différencient d'après la couleur des graines.

C'est une excellente légumineuse fourragère qui peut être récoltée 60 à 80 jours après le semis. Elle résiste à la sécheresse et reste longtemps verte pendant la saison sèche. Elle couvre parfaitement le sol et étouffe toutes les autres plantes.

Elle n'est pas très exigeante quant à la. qualité du sol.

Lé semis se fait en poquets (50 sur $30 \mathrm{~cm}$ ). La floraison commence environ 60 jours après le semis et dure assez longtemps.

Pour l'ensilage, il faut la récolter en septembre, avant que les graines mûrissent. $A$ cette époque, elle fournit environ 10 tonnes de matière verte à l'hectare.

Nous avons obtenu de bons résultats en semant ensemble maìs et Vigna. L'ensilage de ce mélange est de bonne qualité.

Canavalia ensiformis (pois sabre), - Cette légumineuse est originaire de l'Inde. Elle pousse sur toutes les terres.

Elle a une croissance vigoureuse mais assez longue. Son cycle végétatif dure de 5 à 7 mois. Elle donne de grandes gousses $(15 / 25 \mathrm{~cm})$ qui contiennent des graines riches en protides.

On la sème généralement en lignes ou en poquets cspacés do 30 sur $30 \mathrm{~cm}$.

Récoltée à la fin de septembre, elle donne 18 tonnes de matière verte à l'hectare, qu'il est conseillé d'ensiler avec des graminées ou d'autres plantes riches en glucides et pauvres en protides.

Mucuna. - C'est une plante herbacée, rampante, à croissance rapide, et qui résiste bien à la sécheresse. Cultivée pour les graines, elle fournit un bon fourrage vert pour ensilage.

Récoltée au moment de la formation des gousses, elle fournit jusqu'à 20 tonnes à l'hectare.

En mélange avec les plantes riches en glucides, elle donne un ensilage d'excellente qualité et très bien accepté par les animaux.

\section{CONCLUSIONS}

L'ensilage des fourrages est parfaitement possible en zone soudanienne, que l'on utilise des silosfosses ou des silos-meules. Il permet d'apporter aux animaux un complément de ration riche en humidité quand ils ne trouvent en brousse que la paille sèche de médiocre qualité.

L'emploi des silos-meules de 8 à $15 \mathrm{~m}^{3}$ pour ensiler des fourrages spontanés est la méthode recommandable pour les petites exploitations.

L'emploi des silos-tranchées, remplis de graminées cultivées, en mélange avec des légumineuses hachées ou entières, est la méthode recommandable pour les grosses exploitations.

Centre Fédéral de Recherches Zootechniques Bamako (Soudan).

\section{SUMMARY}

\section{Techniques of production of silage in tropical countries.}

1. The cost of production of silage, obtained from mechanically harvested and chopped hay is about $£$ I per ton using a trench silo and a special preservative (Sovilon Solvay) and about $£$ 1.10.0 per ton using formic acid whereas that produced by hand harvesting bush grass and putting it into stacks after having mixed it up with common salt ( $5 \mathrm{~kg} / \mathrm{ton}$ ) is about $£ 1.2 .0$ per ton.

2. Silage obtained by the Sovilon method or the use of formic acid is more appreciated by cattle if sprayed with salt water; the silage produced using common salt is readily eaten by the animals.

3. Stacks silos of 280 to 525 cubic feet are more suitable for small farms using natural hay.

4. On large farms, trench silos filled up with a mixture of cultivated grass and legumes are preferable.

The authors review the advantages and disadvantages of some species of grass which may be used for producing silage. 


\section{RESUMEN}

Nota sobre las técnicas prácticas de fabricación del ensilaje en zona tropical:

La comparación de diversas técnicas de ensilaje ha permitido establecer los hechos siguientes :

1. El coste de fabricación del ensilaje, después de la cosecha del forraje con ayuda de medios mecánicos.y picadura, es aproximadamente de $1.000 \mathrm{fr}$ la tonelada si se emplea un silo-foso y un conservador especial (Sovilon-Solvay); asciende casi a los $1.540 \mathrm{fr}$ si se recurre al acido formico y a $1.120 \mathrm{fr}$, aproximadamente, si se ensila en muela, con sal ordinaria ( $5 \mathrm{~kg} /$ tonelada) de los forrajes de tamojal segados a mano

2. El ganado acepta con mucha más facilidad los ensilajes con Sovilon o con ácido formico rociándolos con agua salada; el ensilaje con sal ordinaria es consumido rápidamente sin ninguna repulsa.

3. Para pequeñas explotaciones que utilizan forrajes espontáneos, parecen preferibles los silos muela de 8 à $15 \mathrm{~m}^{3}$.

4. Para las grandes explotaciones, el método más recomendable es el de silos cortados llenos de una mezcla de gramineas y leguminosas cultivadas.

Algunas especies forrajeras tropicales utilizables para el ensilaje son también revisadas. 